\section{CKD in disadvantaged populations}

"Of all of the forms of inequality, injustice in health is the most shocking and inhumane".

\section{Dr. Martin Luther King, Jr.}

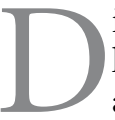
isadvantaged communities, ie, those from low resource areas or households, racial and ethnic minorities, and indigenous and socially disadvantaged backgrounds, have disproportionate burden of unrecognized and untreated chronic kidney disease (CKD). A position of diasadvantage negatively influences healthy behaviors, health care access and environmental exposure, reduces access to goods and services, in particular clean water and sanitation, information about preventive behaviors and adequate nutrition $^{1}$ (Table 1). Low socioeconomic status and poor access to care exacerbate the negative effects of genetic or biologic predisposition.

\section{CKD in developed countries}

Ethnic minorities (African American, Hispanic and Native Americans in the US; South Asians and Afro-caribbeans in the UK and Malays and Indians in Singapore) and indigenous populations (Canadian First Nations people, Australian Aborigines, New Zealand Maoris and Native Americans) experience end stage renal disease (ESRD) at rates 1.5-4 times higher than the general population ${ }^{2}$. In Australia, the increase in the number of indigenous people starting renal replacement therapy (RRT) over the past 25 years exceeded that of the non-indigenous population by 3.5 fold. Particularly remarkable has been the rise in ESRD due to type 2 diabetes, a disease largely attributable to lifestyle issues such as poor nutrition and lack of exercise $^{3}$. Glomerulonephritis and hypertension are also encountered with high frequency in this population.

\section{CKD in developing countries.}

Poverty related factors such as poor sanitation, unsafe water, environmental pollutants and high concentrations of disease-transmitting vectors contribute to the burden of glomerulonephritis and interstitial nephritis in low-income countries. Notable examples include the high prevalence of HIV-associated nephropathy in Sub-Saharan Afri$\mathrm{ca}, \mathrm{CKD}$ related to the use of herbal medicines in Taiwan and Africa, and CKD of unknown etiology in rural agricultural communities from Central America, Egypt, India and Sri Lanka. The latter disproportionately affects male farmworkers and resembles chronic interstitial nephritis in presentation. The etiology remains under investigation, but the implicated factors include exposure to agrochemicals, dehydration, and consumption of high-sucrose drinks or contaminated water. Another distinguishing feature of the ESRD population in developing countries is the relative youth. Many are often part of productive workforce at the time of developing ESRD ${ }^{2}$.

\section{Low-Birth Weight and risk of CKD in the disadvantaged populations}

Maternal malnutrition, leading to low birth weight (LBW) deliveries is more frequent in disadvantaged populations. A correlation between LBW and development of CKD later in life, perhaps linked to low nephron number, has been described in ethnic minorities, indigenous populations and the impoverished. Many of these manifestations, such as hypertension and proteinuria start in childhood and adolescence ${ }^{4}$. Morphometric studies of kidney biopsies in the Aboriginals show glomerulomegaly, perhaps secondary to nephron deficiency, which might predispose to glomerulosclerosis.

\section{Disparities in Access to Renal Replacement Therapy}

In 2010, about 2.6 million people were on dialysis worldwide, $93 \%$ in high or upper middleincome countries. By contrast, conservative estimates placed the number of people requiring RRT at 4.9 million, suggesting that at least 2.3 million died prematurely because of lack of access to RRT. The current provision of RRT was linked strongly to per capita GNP, suggesting that poverty is a major disadvantage for receiving RRT. The relationship between income and access to $\mathrm{RRT}$ is almost linear in low-income countries. In 
Table 1. Possible mechanisms by which poverty increases the burden of disease

\begin{tabular}{|c|c|c|c|}
\hline Health Behavior & Access to Health Care & Biological Factors & Enviromental Factors \\
\hline $\begin{array}{l}\text { - Lack of information on } \\
\text { preventive behaviors } \\
\text { - Lack of knowledge on } \\
\text { how best to respond to } \\
\text { an episode of illness } \\
\text { - Health beliefs and un- } \\
\text { healthy behavior }\end{array}$ & $\begin{array}{l}\text { - Lack of access to health } \\
\text { care } \\
\text { - Greater distance from } \\
\text { health care providers } \\
\text { - Lack of out-of- pocket } \\
\text { resources }\end{array}$ & $\begin{array}{l}\text { - Low-birth weight } \\
\text { - Genetic predisposition } \\
\text { - Cumulative biological risk } \\
\text { profiles } \\
\text { - Inadequate nutrition }\end{array}$ & $\begin{array}{l}\text { - Increased exposure to } \\
\text { pollutants } \\
\text { - Increased exposure to } \\
\text { communicable diseases } \\
\text { - Lack of clean water and } \\
\text { sanitation }\end{array}$ \\
\hline
\end{tabular}

some parts of the world, less than $10 \%$ of eligible ESRD patients receive RRT. By 2030, the number of people receiving RRT globally is projected to increase to 5.4 million. Most of this increase will be in developing countries of Asia and Africa ${ }^{2}$. Poor countries have also low transplant rates because of a combination of low levels of infrastructure; geographical remoteness; lack of legislation governing brain death; religious, cultural and social constraints; and commercial incentives that favour dialysis ${ }^{5}$. Even in developed countries like Australia, New Zealand, UK and USA where dialysis is widely available, access has been shown to be disproportionately low amongst indigenous and ethnic minority groups. In particular, they are less likely to receive home-based therapies such as peritoneal dialysis (PD) and home hemodialysis and have lower rates of kidney transplantation.

Socioeconomic disparities and fragmentation of healthcare system have significant impact on the delivery of RRT within developing countries, as shown by data from India and Mexico. Living in rural areas, extremes of age, female gender and lack of insurance or reimbursement reduce the likelihood of getting RRT. Furthermore, the relationship between poverty and CKD is bidirectional. ESRD treatment costs are often met by out-of-pocket expenditure, which pushes families deeper into poverty, and impacts national productivity $^{2}$.

\section{Outcomes}

Overall treatment outcomes are relatively poor among the indigenous, ethnic minorities, and uninsured populations who do receive RRT, even after adjustment for co-morbidities. These measures include mortality rates; achievement of target levels for blood pressure, mineral metabolism and nutrition; time to transplantation; and technique failure on peritoneal dialysis.

\section{Summary}

The aforementioned places a special burden on the professional community and policymakers to ensure provision of appropriate renal care to the disadvantaged populations. This will require both supply and demand side actions: expanding the reach of dialysis through development of lowcost alternatives that can be practised in remote locations, and implementation and evaluation of cost-effective prevention strategies. Kidney transplantation should be promoted by expanding deceased donor transplant programs and use of inexpensive, generic immunosuppressive drugs. The message of WKD 2015 is that a concerted attack against the diseases that lead to ESRD, by increasing community outreach, better education, improved economic opportunity, and access to preventive medicine for those at highest risk, could end the unacceptable relationship between CKD and disadvantage in these communities.

\section{References}

1. Sachs JD. Macroeconomics and health: Investing in health for economic development. Report of the Commission on Macroeconomics and Health. WHO. 2001.

2. Jha V, García-García G, Iseki K, Li Z, Naicker S, Plattner 
B, et al. Chronic kidney disease: global dimension and perspectives. Lancet 2013; 382: 260-72.

3. Collins JF. Kidney disease in Maori and Pacific people in New Zealand. Clin Nephrol 2010; 74 Suppl. 1: S61-5.

4. Hoy WE, Hughson MD, Zimanyi M, et al. Distribution of volume of individual glomeruli in kidneys at autopsy: association with age, nephron number, birthweight and body mass index. Clin Nephrol 2010; 74 Suppl 1: S10512.

5. García-García, Harden PN, Chapman JR. World Kidney Day 2012. The Global role of kidney transplantation. The Lancet 2012; 379: e36-8.
Guillermo García-García ${ }^{1}$ Vivekanand $\mathrm{Jh}^{2}$ on behalf of the World Kidney Day Steering Committee ${ }^{1}$ Nephrology Service, Hospital Civil de Guadalajara

University of Guadalajara Health Sciences Center Guadalajara, Jal. Mexico.

${ }^{2}$ Postgraduate Institute of Medical Education and Research, Chandigarh, India, George Institute for Global Health, New Delhi, India and University of Oxford, UK.

Correspondence to:

World Kidney Day, International Society of Nephrology.

Rues de Fabriques 1B, 1000, Brussels, Belgium

info@worldkidneyday.org

*Members of the World Kidney Day Steering Committee are: Philip Kam Tao Li, Guillermo García-García, William G. Couser, Timur Erk, Elena Zakharova, Luca Segantini, Paul Shay, Miguel C. Riella, Charlotte Osafo, Sophie Dupuis, Charles Kernahan. 